\title{
Endovascular Salvage of a False Aneurysm of the Posterior Tibial Artery Caused by a Stab From a Stingray
}

\author{
E. J. van Helden $\cdot$ D. Eefting $\cdot$ J. Florie $\cdot$ \\ H. J. M. Verhagen $\cdot$ A. Moelker
}

Received: 15 October 2013/Accepted: 17 November 2013/Published online: 19 December 2013

(C) Springer Science+Business Media New York and the Cardiovascular and Interventional Radiological Society of Europe (CIRSE) 2013

\section{Case Report}

A 28-year-old woman was referred to our tertiary hospital with persistent tenderness and edema of the right lower leg after receiving a stab from a stingray. She had no past medical history and was not on any medication.

Two months before her referral, she had traveled to the Republic of Seychelles. While bathing in the sea, she was stabbed by a stingray in the right lower leg. At that time she was treated with oral antibiotics (cloxacillin) and oral prednisolone. Due to persistent pain, swelling, and discoloration of her calf, she was treated surgically in a regional hospital with local superficial soft tissue excision. Tissue cultures were negative, and microscopic examination showed a foreign body, most likely a piece of the tail of the stingray. The patient experienced persisting pain in her lower leg. Duplex ultrasound examination showed a large $(43 \times 25 \mathrm{~mm})$ false aneurysm of the posterior tibial artery

\author{
E. J. van Helden - D. Eefting · J. Florie . \\ H. J. M. Verhagen - A. Moelker \\ Erasmus Medical Centre, 's-Gravendijkwal 230, \\ 3015 CE Rotterdam, The Netherlands \\ e-mail: d.eefting@erasmusmc.nl \\ J. Florie \\ e-mail: j.florie@erasmusmc.nl \\ H. J. M. Verhagen \\ e-mail: h.verhagen@erasmusmc.nl \\ A. Moelker \\ e-mail: a.moelker@erasmusmc.nl \\ E. J. van Helden ( $₫)$ \\ Nieuwe Crooswijkseweg 60B, 3034 PS Rotterdam, \\ The Netherlands \\ e-mail: erikvanhelden87@gmail.com
}

(PTA). She was referred to our hospital for further treatment.

On physical examination, she had a slightly swollen painful right lower leg with a well-healing surgical wound distally on the dorsolateral side. On palpation, a clear thrill was present over the skin defect. A computed tomography (CT)-angiography of the right lower leg showed a large false aneurysm of the PTA. In addition, an arteriovenous fistula (AVF) had developed between the aneurysm and the posterior tibial vein (Figs. 1, 2).

Because this aneurysm had a broad base and an AVF had formed, direct thrombin injection was not preferable due to the presumed higher risk of intravascular thrombosis and distal embolization. We decided to close the base of the false aneurysm with a $3 \times 30 \mathrm{~mm}$ PTA balloon (Ultra Soft Monorail, Boston Scientific International, Nanterre, France; inflated during $<2$ min with a pressure of just above $1 \mathrm{~atm}$ ) inserted through a 5F sheath after antegrade puncture of the common femoral artery (Fig. 3A). To prevent reflux of thrombin (D-stat Vascular Solutions, Galway, Ireland) into the venous system, a tourniquet was applied. Subsequently, the false aneurysm was punctured under ultrasound and fluoroscopic guidance, and thrombin ( 5,000 U; Tissuecol, Baxter) mixed with iodine contrast agent was injected into the aneurysm. This procedure was performed with heparin (3.000 U) infusing over the sheath. Angiography directly after embolization showed an open PTA, minimal opacification of the aneurysm at the arterial site, and no signs of an AVF or intimal injury (Fig. 3B). The postprocedural duplex ultrasound 1 day later showed no flow in the false aneurysm with normal flow through the PTA. The patient's recovery was uneventful, and was she discharged with no complaints. After 8 months, duplex ultrasound showed a completely thrombosed aneurysm and a decrease in the size of the aneurysm ( 24-mm maximum diameter). 


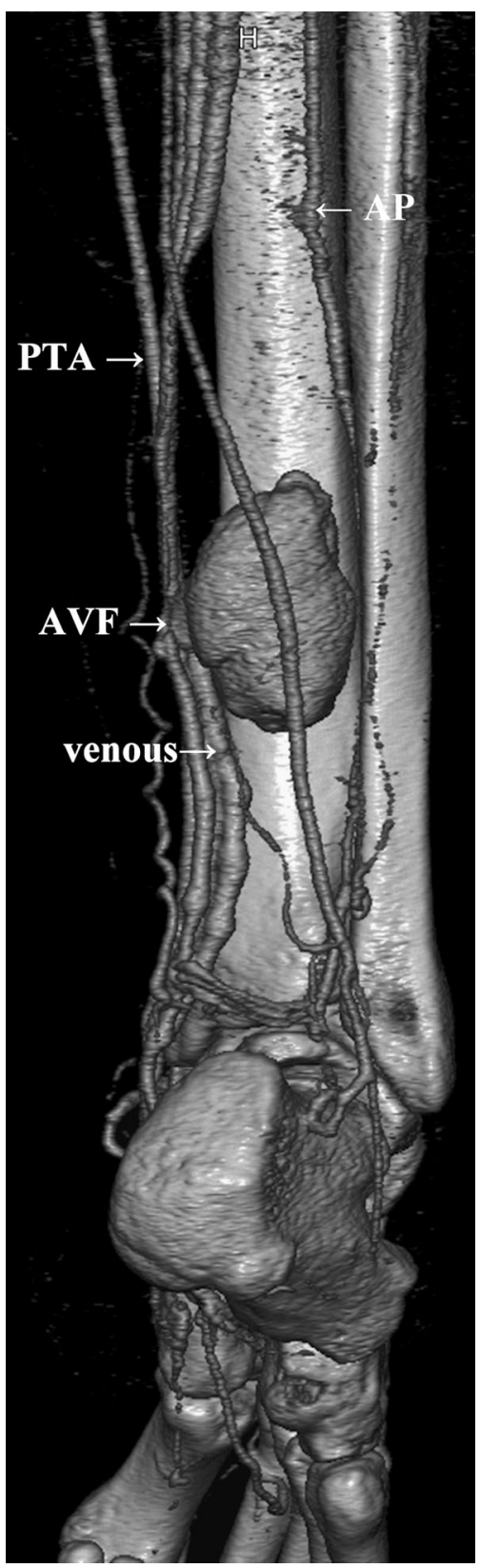

Fig. 1 False aneurysm of the PTA with a broad base, AVF and venous congestion

Unfortunately the PTA was occluded at the site of the aneurysm. Distally the artery was open with filling by way of collaterals. The patient had no clinical symptoms and unimpaired walking distance.

\section{Discussion}

False aneurysms of the tibial arteries are rare, and the pathophysiology is often of traumatic or iatrogenic origin. Causes may be penetrating vessel trauma, orthopaedic

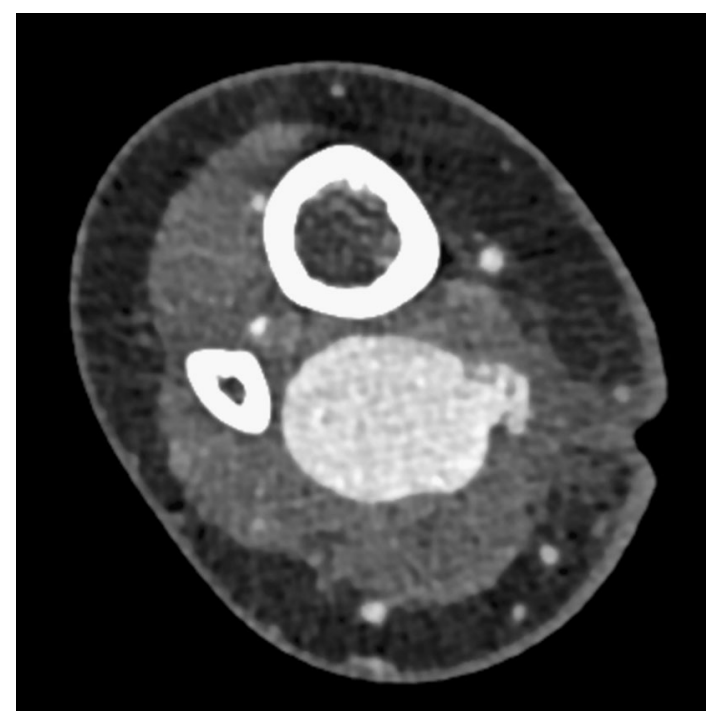

Fig. 2 Underneath the soft tissue defect is the false aneurysm and the fistula with artery and vein are showing

procedures, endovascular surgery, and graft anastomosis [1-5]. Small false aneurysms are often asymptomatic and can sometimes regress completely when thrombosis occurs. However, if they progressively increase in volume, adjacent structures, such as nerves, arteries, and veins, could obtain pinched, thus swelling, pain, and paresthesia [2].

Treatment for false aneurysms is traditionally performed by open surgical technique consisting of exploration and ligation or arterial repair with vein patch or interposition grafting. Disadvantages are the collateral damage and complications, such as infection, bleeding, and anesthetic complications. Less invasive alternatives are duplex-guided compression, thrombin injection, or an endovascular intervention, such as coiling or stenting [1]. We considered using a small $3.0 \mathrm{~mm}$-diameter covered stent. However, this could entail lifelong use of anticoagulants, greater risk of reintervention, and longer postoperative follow-up. In addition, little is known about the long-term patency of a covered sent in the tibial artery. For those reasons, we decided that stenting was not a preferable option $[6,7]$.

Thrombin injection is a safe and quick method; however, in this case we reasoned that the risk of intravascular thrombosis and distal arterial embolization would be greater due to the broad base of the false aneurysm [8]. Therefore, we chose to occlude the base of the aneurysm with a 3.0-mm percutaneous transluminal angioplasty balloon before the thrombin injection, thus minimizing intravascular spill of thrombin. Unfortunately, the PTA eventually occluded. This occlusion, which was not observed at 1 day after the procedural, might have been due to gradual growth and protrusion of the thrombus into the native vessel. 
Fig. 3 A Preprocedural; showing the PTA and the aneurysm. B Postprocedural; the catheter is still in the PTA, overlapping the base of the false aneurysm and the AVF. The needle, intended for thrombin injections, is still in the aneurysm. Notice the minimal opacification of the aneurysm and the open artery
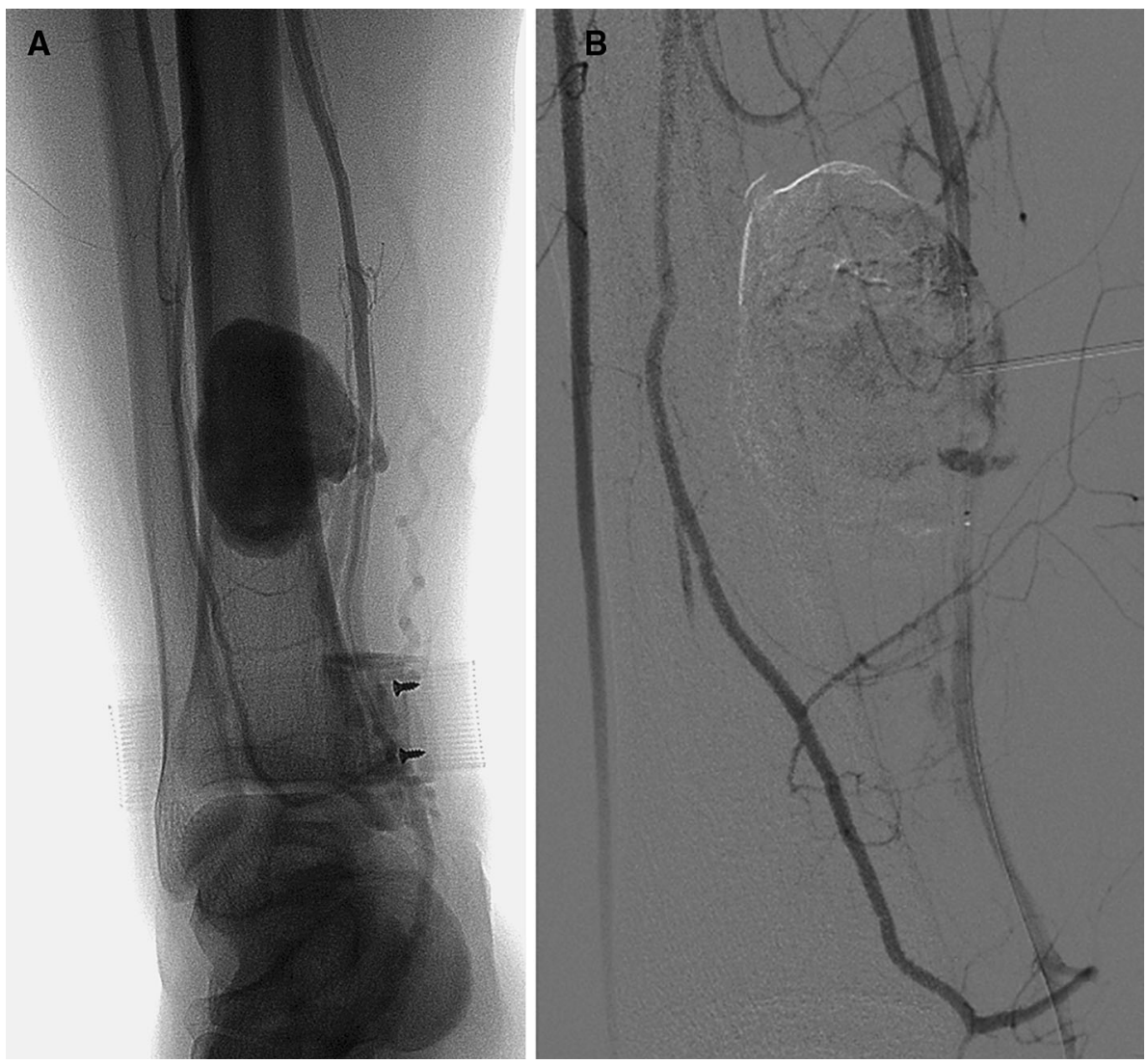

\section{Conclusion}

Thrombin injection is a good alternative to open surgery for treating false aneurysms of the crural arteries in selected cases and has its own specific (dis)advantages.

Conflict of interest No potential conflicts of interest were disclosed.

\section{References}

1. Joglar F, Kabutey NK, Maree A, Farber A (2010) The role of stent grafts in the management of traumatic tibial artery pseudoaneurysms: case report and review of the literature. Vasc Endovasc Surg 44(5):407-409

2. Bartoli MA, Lerussi GB, Gulino R, Schroeder M, Branchereau A (2010) False aneurysm at the origin of the anterior tibial artery after opening wedge osteotomy. Vascular 18(1):45-48
3. Fraser JD, Cully BE, Rivard DC, Leys CM, Holcomb GW, St Peter SD (2009) Traumatic pseudoaneurysm of the anterior tibial artery treated with ultrasound-guided thrombin injection in a pediatric patient. J Pediatr Surg 44(2):444-447

4. De Roo RA, Steenvoorde P, Schuttevaer MH, den Outer AJ, Oskam J, Hedeman Joosten PP (2004) Exclusion of a crural pseudoaneurysm with a PTFE-covered stent-graft. J Endovasc Ther 11:344-347

5. Zelent ME, Neese DJ (2009) Posterior tibial artery pseudoaneurysm identified subsequent to surgical wound dehiscence. J Foot Ankle Surg 48:56-60

6. Garg K, Rockman CB, Kim BJ, Jacobowitz GR, Maldonado TS, Adelman MA et al (2012) Outcome of endovascular repair of popliteal artery aneurysm using the Viabahn endoprosthesis. J Vasc Surg 55:1647-1653

7. Joglar F, Kabutey NK, Maree A, Farber A (2010) The role of stent grafts in the management of traumatic tibial artery pseudoaneurysms: case report and review of the literature. Vasc Endovasc Surg 44:407

8. Hoffmann I, Wunderlich N, Robertson G, Kieback A, Haller C, Pfeil W et al (2007) Percutaneous injection of thrombin for the treatment of pseudoaneurysms: the German multicentre registry. Eurointervention 3:321-326 